Article

\title{
New Refinements and Improvements of Jordan's Inequality
}

\author{
Lina Zhang ${ }^{1}$ and Xuesi Ma ${ }^{2, *}$ \\ 1 School of Computer Science and Technology, Henan Polytechnic University, Jiaozuo 454000, China; \\ zln@hpu.edu.cn \\ 2 School of Mathematic and Information Science, Henan Polytechnic University, Jiaozuo 454000, China \\ * Correspondence: maxuesi@hpu.edu.cn
}

Received: 8 November 2018; Accepted: 21 November 2018; Published: 26 November 2018

\begin{abstract}
The polynomial bounds of Jordan's inequality, especially the cubic and quartic polynomial bounds, have been studied and improved in a lot of the literature; however, the linear and quadratic polynomial bounds can not be improved very much. In this paper, new refinements and improvements of Jordan's inequality are given. We present new lower bounds and upper bounds for strengthened Jordan's inequality using polynomials of degrees 1 and 2. Our bounds are tighter than the previous results of polynomials of degrees 1 and 2. More importantly, we give new improvements of Jordan's inequality using polynomials of degree 5 , which can achieve much tighter bounds than those previous methods.
\end{abstract}

Keywords: Jordan's inequality; polynomial; bound

\section{Introduction}

The following inequality

$$
\frac{2}{\pi} \leq \operatorname{sinc}(x)=\frac{\sin x}{x}<1, \quad x \in(0,2 / \pi],
$$

with equality holds if and only if $x=\pi / 2$, is the famous Jordan's inequality [1]. $\operatorname{sinc}(x)$ is also called the "sampling function" that arises frequently in signal processing and the theory of Fourier transforms. The Jordan's inequality plays an important role in many areas of pure and applied mathematics. Many improvements and refinements of Jordan's inequality were presented in the recent period [2-7]. There are some sharp lower and upper bounds for the $\operatorname{sinc}(x)$ function by using polynomial degrees from 1 to 4 .

Zhang et al. [8] gave that

$$
\frac{2}{\pi}+\frac{\pi-2}{\pi^{2}}(\pi-2 x) \leq \frac{\sin x}{x} \leq \frac{2}{\pi}+\frac{2}{\pi^{2}}(\pi-2 x), \quad x \in(0,2 / \pi] .
$$

Qi et al. [9] proved that

$$
\frac{2}{\pi}+\frac{1}{\pi^{3}}\left(\pi^{2}-4 x^{2}\right) \leq \frac{\sin x}{x} \leq \frac{2}{\pi}+\frac{\pi-2}{\pi^{3}}\left(\pi^{2}-4 x^{2}\right), \quad x \in(0,2 / \pi] .
$$

Deng [10] presented

$$
\frac{2}{\pi}+\frac{2}{3 \pi^{4}}\left(\pi^{3}-8 x^{3}\right) \leq \frac{\sin x}{x} \leq \frac{2}{\pi}+\frac{\pi-2}{\pi^{4}}\left(\pi^{3}-8 x^{3}\right), \quad x \in(0,2 / \pi],
$$


and Jiang et al. [11], similarly, gave the result

$$
\frac{2}{\pi}+\frac{1}{2 \pi^{5}}\left(\pi^{4}-16 x^{4}\right) \leq \frac{\sin x}{x} \leq \frac{2}{\pi}+\frac{\pi-2}{\pi^{5}}\left(\pi^{4}-16 x^{4}\right), \quad x \in(0,2 / \pi]
$$

Equalities in Labels (2)-(5) are valid if and only if $x=\pi / 2$. As $x \rightarrow 0^{+}$, the equalities on the right-hand sides of (3)-(5) are valid, but strict inequalities on the left-hand sides of (3)-(5) and two sides of (2) persist. Debnath et al. [12] gave the improvements of (3) and (5)

$$
g_{4, D 1}^{l}(x) \leq \frac{\sin x}{x} \leq g_{4, D 1}^{u}(x), \quad x \in(0,2 / \pi]
$$

and

$$
g_{4, D 2}^{l}(x) \leq \frac{\sin x}{x} \leq g_{4, D 2}^{u}(x), \quad x \in(0,2 / \pi]
$$

where $g_{4, D 1}^{l}(x)=\frac{2}{\pi}+\frac{1}{\pi^{3}}\left(\pi^{2}-4 x^{2}\right)+\left(1-\frac{3}{\pi}\right)-\left(\frac{1}{6}-\frac{4}{\pi^{3}}\right) x^{2}, g_{4, D 1}^{u}(x)=\frac{2}{\pi}+\frac{1}{\pi^{3}}\left(\pi^{2}-4 x^{2}\right)+(1-$ $\left.\frac{3}{\pi}\right)-\left(\frac{1}{6}-\frac{4}{\pi^{3}}\right) x^{2}+\frac{1}{120} x^{4}, g_{4, D 2}^{l}(x)=\frac{2}{\pi}+\frac{1}{2 \pi^{5}}\left(\pi^{4}-16 x^{4}\right)+\left(1-\frac{5}{2 \pi}\right)-\frac{1}{6} x^{2}, g_{4, D 2}^{u}(x)=\frac{2}{\pi}+\frac{\pi-2}{2 \pi^{5}}\left(\pi^{4}-\right.$ $\left.16 x^{4}\right)+\left(1-\frac{5}{2 \pi}\right)-\frac{1}{6} x^{2}+\left(\frac{8}{\pi^{5}}+\frac{1}{120}\right) x^{4}$.

As $x \rightarrow 0^{-}$, equalities on two sides of (6) and (7) are valid; however, as $x \rightarrow \frac{\pi}{2}^{-}$, the lower and upper limits of (6) and (7) are different from that of $\operatorname{sinc}(x)$. The problem of strict inequalities of (6) and (7) still exists.

In order to ensure that the equality of Jorand's inequality is valid near zero and $\pi / 2$, Agarwal et al. [13] and Chen et al. [14] gave new lower and upper bounds by using polynomials of degree of 3 and 4 ,

$$
\begin{gathered}
g_{3, A}^{l}(x) \leq \frac{\sin x}{x} \leq g_{3, A}^{u}(x), \quad x \in(0,2 / \pi] \\
g_{3, C}^{l}(x) \leq \frac{\sin x}{x} \leq g_{3, C}^{u}(x), x \in(0,2 / \pi], \\
g_{4, C}^{l}(x) \leq \frac{\sin x}{x} \leq g_{4, C}^{u}(x), \quad x \in(0,2 / \pi),
\end{gathered}
$$

where $g_{3, A}^{l}(x)=1+\frac{4\left(66-43 \pi+7 \pi^{2}\right)}{\pi^{2}} x-\frac{4\left(124-83 \pi+14 \pi^{2}\right)}{\pi^{3}} x^{2}-\frac{4(12-4 \pi)}{\pi^{4}} x^{3}, g_{3, A}^{u}(x)=1+$ $\frac{4\left(75-49 \pi+8 \pi^{2}\right)}{\pi^{2}} x-\frac{4\left(142-95 \pi+16 \pi^{2}\right)}{\pi^{3}} x^{2}-\frac{4(12-4 \pi)}{\pi^{4}} x^{3}, g_{3, C}^{l}(x)=1-\frac{4(3 \pi-8)}{\pi^{3}} x^{2}+\frac{16(\pi-3)}{\pi^{4}} x^{3}, g_{3, C}^{u}(x)=$ $1-\frac{2(5 \pi-2-16 \sqrt{2}+2 \sqrt{2} \pi)}{\pi^{2}} x+\frac{8(4 \pi-4-16 \sqrt{2}+3 \sqrt{2} \pi)}{\pi^{3}} x^{2}-\frac{32(\pi-2-4 \sqrt{2}+\sqrt{2} \pi)}{\pi^{4}} x^{3}, g_{4, \mathrm{C}}^{l}(x)=1-$ $\frac{4(-48 \sqrt{2}-2+17 \pi+4 \sqrt{2} \pi)}{\pi^{3}} x^{2}+\frac{32(-28 \sqrt{2}-2+9 \pi+3 \sqrt{2} \pi)}{\pi^{4}} x^{3}-\frac{64(-16 \sqrt{2}-2+5 \pi+2 \sqrt{2} \pi)}{\pi^{5}} x^{4}, g_{4, C}^{u}(x)=1-$ $\frac{4(-8 \sqrt{2}-7+3 \pi+2 \sqrt{2} \pi)}{\pi^{2}} x+\frac{4(-32 \sqrt{2}-68+13 \pi+16 \sqrt{2} \pi)}{\pi^{3}} x^{2}-\frac{32(-4 \sqrt{2}-26+3 \pi+5 \sqrt{2} \pi)}{\pi^{4}} x^{3}+\frac{64(-12+\pi+2 \sqrt{2} \pi)}{\pi^{5}} x^{4}$.

Zeng and $\mathrm{Wu}[15]$ gave the polynomial bounds of degree $m(m \geq 2)$ for $\operatorname{sinc}(\mathrm{x})$

$$
\frac{2}{\pi}+\frac{2}{m \pi^{m+1}}\left(\pi^{m}-2^{m} x^{m}\right) \leq \frac{\sin x}{x} \leq \frac{2}{\pi}+\frac{\pi-2}{\pi^{m+1}}\left(\pi^{m}-2^{m} x^{m}\right), \quad x \in(0,2 / \pi] .
$$

Putting $m=2,3,4$ in (11) results in (3), (4) and (5), respectively.

The cubic and quartic polynomial lower and upper bounds of $\operatorname{sinc}(x)$ have been improved in a lot of literature; however, the linear and quadratic polynomial lower and upper bounds can not be improved very well. To give new tighter linear and quadratic polynomial bounds is the first aim of the paper. The second aim is to further refine and generalize the Jordan's inequality. 
The paper gives improvements of the polynomial bounds of degrees 1 and 2. More importantly, we present new improvements of Jordan's inequality using polynomials of degree 5, which can achieve much tighter bounds than those previous methods.

\section{Results}

In this section, we will give some results about the $\mathrm{n}$-th-order derivative and two-sides bounds of $\operatorname{sinc}(x)$. Firstly, we present a Lemma that is very useful for our proof [16].

Lemma 1. Let $w_{0}, w_{1}, \cdots, w_{r}$ be $r+1$ distinct points in $[a, b]$, and $n_{0}, n_{1}, \cdots n_{r}$ be $r+1$ integers $\geq 0$. Let $N=n_{0}+\cdots+n_{r}+r$. Suppose that $g(t)$ is a polynomial of degree $N$ such that

$$
g^{(i)}\left(w_{j}\right)=f^{(i)}\left(w_{j}\right), \quad i=0, \cdots, n_{j}, j=0, \cdots, r .
$$

Then, there exists $\xi(t) \in[a, b]$ such that

$$
f(t)-g(t)=\frac{f^{(N+1)}(\xi(x))}{(N+1) !} \prod_{i=0}^{r}\left(t-w_{i}\right)^{n_{i}+1} .
$$

Next, we give Theorems of n-th-order derivative and two sides bounds of $\operatorname{sinc}(x)$ using polynomials of degrees 1 and 2.

Theorem 1. For $x \in(0, \pi / 2]$, we have

$$
\operatorname{sinc}^{(n)}(x)=\frac{f_{n}(x)}{x^{n+1}}
$$

where $f_{n}(x)=x f_{n-1}^{\prime}(x)-n f_{n-1}(x), f_{n}^{\prime}(x)=-x^{n} \sin \left(x+\frac{n \pi}{2}\right), f_{1}(x)=-\sin (x)+x \cos (x)$, and $\operatorname{sinc}^{(n}(x)$ denotes the $n$-th-order derivative of $\operatorname{sinc}(x)$.

Proof. For the definition of $\operatorname{sinc}(x)$, we have

$$
\operatorname{sinc}^{\prime}(x)=\frac{-\sin (x)+x \cos (x)}{x^{2}}
$$

then, $f_{1}(x)=-\sin (x)+x \cos (x), f_{1}^{\prime}(x)=-x \sin (x)$.

Let $\operatorname{sinc}^{(m)}(x)=\frac{f_{m}(x)}{x^{m+1}}=\frac{x f_{m-1}^{\prime}(x)-m f_{m-1}(x)}{x^{m+1}}, f_{m}^{\prime}(x)=-x^{m} \sin \left(x+\frac{m \pi}{2}\right)$; then, as $n=m+1$,

$$
\begin{gathered}
\operatorname{sinc}^{(m+1)}(x)=\frac{d}{d x} \operatorname{sinc}^{(m)}(x)=\frac{d}{d x} \frac{f_{m}(x)}{x^{m+1}}=\frac{x f_{m}^{\prime}(x)-(m+1) f_{m}(x)}{x^{m+2}}=\frac{f_{m+1}(x)}{x^{m+2}}, \\
f_{m+1}^{\prime}(x)=x f_{m}^{\prime \prime}(x)-m f_{m}^{\prime}(x)=-x^{(m+1)} \sin \left(x+\frac{(m+1) \pi}{2}\right) .
\end{gathered}
$$

The proof of Theorem 1 is completed.

Theorem 2. For $x \in(0, \pi / 2]$,

$$
1+\frac{4-2 \pi}{\pi^{2}} x \leq \frac{\sin x}{x} \leq \frac{8 \sqrt{2}-\sqrt{2} \pi}{2 \pi}+\frac{2 \sqrt{2}-8 \sqrt{2}}{\pi^{2}} x .
$$

Proof. Let $b=\pi / 2, c=\pi / 4, e_{1, l}(x)=\operatorname{sinc}(x)-1-\frac{4-2 \pi}{\pi^{2}} x$, and $e_{1, u}(x)=\operatorname{sinc}(x)-\frac{8 \sqrt{2}-\sqrt{2} \pi}{2 \pi}+$ $\frac{2 \sqrt{2}-8 \sqrt{2}}{\pi^{2}} x$, for $x \in(0, \pi / 2]$, we have

$$
e_{1, l}(0)=e_{l, 1}(b)=0, \quad e_{1, u}(c)=e_{1, u}^{\prime}(c)=0 .
$$


By Lemma 1 and Theorem $1, e_{1, l}^{\prime \prime}(x)=e_{u, 1}^{\prime \prime}(x)=\operatorname{sinc}^{\prime \prime}(x) \leq 0$, and there exists $\xi_{j}(x) \in[0, \pi / 2]$, $j=1,2$, such that

$$
e_{1, l}(x)=e_{l, 1}^{\prime \prime}\left(\xi_{1}(x)\right) x(x-b) \geq 0, \quad e_{1, u}(x)=e_{1, u}^{\prime \prime}\left(\xi_{2}(x)\right)(x-c)^{2} \leq 0
$$

The proof is finished.

Theorem 3. For $x \in(0, \pi / 2]$,

$$
g_{2}^{l}(x) \leq \frac{\sin x}{x} \leq g_{2}^{u}(x)
$$

where $g_{2}^{l}(x)=1+\frac{12-4 \pi}{\pi^{2}} x+\frac{4 \pi-16}{\pi^{3}} x^{2}, g_{2}^{u}=1+\frac{8-4 \pi}{\pi^{3}} x^{2}$.

Proof. Let $b=\pi / 2, e_{2, l}(x)=\operatorname{sinc}(x)-g_{2}^{l}(x)$,

$$
e_{2, l}(0)=e_{2, l}(b)=e_{2, l}^{\prime}(b)=0, \quad e_{2, u}(0)=e_{2, u}(b)=e_{2, u}^{\prime}(0)=0 .
$$

By Lemma 1 and Theorem $1, e_{1, l}^{(3)}(x)=e_{u, 1}^{(3)}(x)=\operatorname{sinc}^{(3)}(x) \geq 0$, and there exists $\xi_{j}(x) \in$ $[0, \pi / 2], j=3,4$, such that

$$
e_{2, l}(x)=e_{2, l}^{(3)}(\xi(x)) x(x-b)^{2} \geq 0, \quad e_{2, u}(x)=e_{2, u}^{(3)}(\xi(x)) x^{2}(x-b) \leq 0
$$

The proof is finished.

Theorems 2 and 3 give new bounds of $\operatorname{sinc}(x)$ using polynomials of degrees 1 and 2. Figures 1 and 2 give the error between $\operatorname{sinc}(x)$ and the polynomial bounds from degree 1 to 2 . Both figures show that our bounds are tighter than the previous results. The same conclusion can also be shown in Table 1. Error low and Error upp $_{\text {lon }}$ denote the maximum errors between $\operatorname{sinc}(x)$ and the lower and upper bounds, respectively. It is obvious that the maximum errors are less than or equal to those of previous methods using polynomials of degrees 1 and 2.

Table 1. Maximum errors from different methods.

\begin{tabular}{ccc}
\hline \multirow{2}{*}{ Method } & \multicolumn{2}{c}{ Error } \\
\cline { 2 - 3 } & Error $_{\text {low }}$ & Error $_{\text {up }}$ \\
\hline Linear polynomial (Equation (2)) & 0.08239552616791 & 0.27319901792837 \\
Linear polynomial (Equation (12)) & 0.08239552616791 & 0.09343987891909 \\
Quadratic polynomial (Equation (3)) & 0.04507034107202 & 0.01161202091677 \\
Quadratic polynomial (Equation (13)) & 0.01541234761755 & 0.01161202091677 \\
Cubic polynomial (Equation (4)) & 0.15117363517661 & 0.06535850279048 \\
Cubic polynomial (Equation (8)) & 0.00263153345090 & 0.00098638493116 \\
Cubic polynomial (Equation (9)) & 0.18004172509621 & 0.00065651979512 \\
Quartic polynomial (Equation (5)) & 0.20422528287386 & 0.10245473620764 \\
Quartic polynomial (Equation (6)) & 0.04777095540497 & 0.00287304555420 \\
Quartic polynomial (Equation (7)) & 0.20664387547518 & 0.20422528454052 \\
Quartic polynomial (Equation (10)) & 0.00010491761462 & 0.00011278132149 \\
Quintic Polynomial (Equation (11) $\mathrm{m}=5$ ) & 0.236056271492236 & 0.129868221438454 \\
Quintic Polynomial (Equation (14)) & $\mathbf{0 . 0 0 0 0 1 0 5 9 9 8 9 6 2 2}$ & $\mathbf{0 . 0 0 0 0 0 5 4 5 6 2 8 3 4 3}$ \\
Octic Polynomial (Equation (11) m =8) & 0.283802754419804 & 0.182471527648186 \\
Decic Polynomial (Equation (11) $\mathrm{m}=10$ ) & 0.299718248728994 & 0.204647143136328 \\
\hline
\end{tabular}

Theorem 4. For $x \in(0, \pi / 2]$,

$$
g_{5}^{l}(x) \leq \frac{\sin x}{x} \leq g_{5}^{u}(x)
$$


where $g_{5}^{l}(x)=1+\frac{32-2048 \sqrt{2}+2187 \sqrt{3}-(113+128 \sqrt{2}) \pi}{2 \pi^{2}} x+\frac{-448+26624 \sqrt{2}-27702 \sqrt{3}+(1255+1536 \sqrt{2}) \pi}{2 \pi^{3}} x^{2}$

$+\frac{1168-62464 \sqrt{2}+64152 \sqrt{3}-(2825+3392 \sqrt{2}) \pi}{\pi^{4}} x^{3}+\frac{-2688+125952 \sqrt{2}-128304 \sqrt{3}+(5664+6528 \sqrt{2}) \pi}{\pi^{5}} x^{4}$

$+\frac{2304-92160 \sqrt{2}+93312 \sqrt{3}-(4176+4608 \sqrt{2}) \pi}{\pi^{6}} x^{5}, \quad g_{5}^{u}(x)=1+\frac{64+256 \sqrt{2}-(92+32 \sqrt{2}) \pi}{\pi^{3}} x^{2}+$ $\frac{-624-1536 \sqrt{2}+(528+256 \sqrt{2}) \pi}{\pi^{4}} x^{3}$

$+\frac{1920+3072 \sqrt{2}-(1088+640 \sqrt{2}) \pi}{\pi^{5}} x^{4}+\frac{-1792-2048 \sqrt{2}+(768+512 \sqrt{2}) \pi}{\pi^{6}} x^{5}$.

Proof. Let $b=\frac{\pi}{2}, c=\frac{\pi}{4}, d=\frac{\pi}{3}, e_{5, l}(x)=\operatorname{sinc}(x)-g_{5}^{l}(x), e_{5, u}(x)=\operatorname{sinc}(x)-g_{5}^{u}(x)$. It is obvious that for $x \in(0, \pi / 2], e_{5, l}^{(6)}(x)=e_{5, u}^{(6)}(x)=\operatorname{sinc}^{(6)}(x)$.

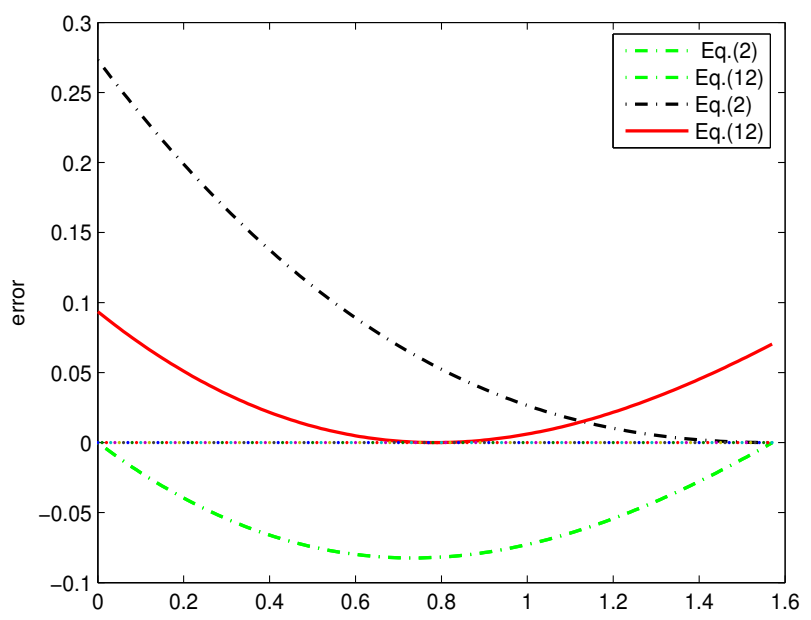

Figure 1. Error plots between $\operatorname{sinc}(x)$ and the bounds of Equations (2) and (12).

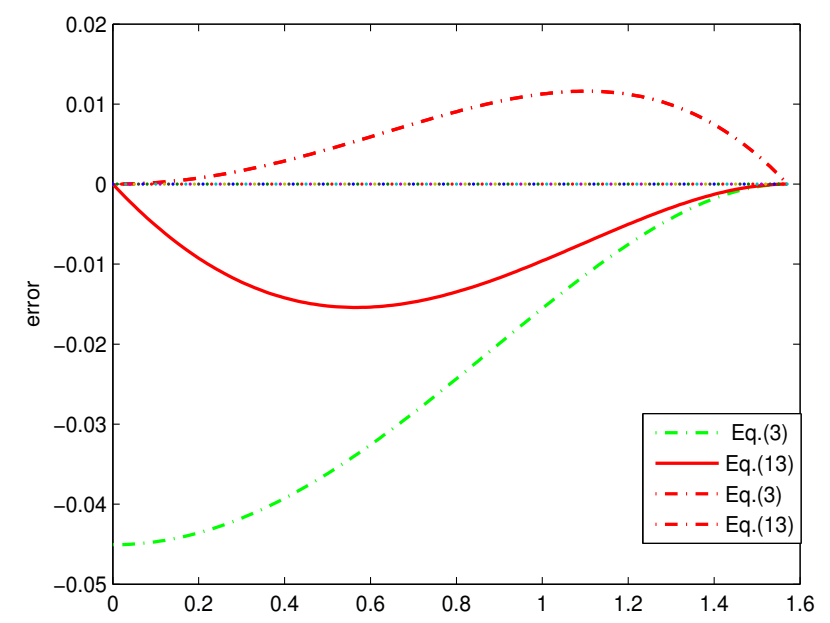

Figure 2. Error plots between $\operatorname{sinc}(x)$ and the bounds of Equations (3) and (13).

By Theorem 1 and Lemma 1 , we have $\operatorname{sinc}^{(6)}(x) \leq 0$ and there exists $\varepsilon \in(0, \pi / 2]$ such that

$$
\begin{gathered}
e_{5, l}(0)=e_{5, l}(b)=e_{5, l}(c)=e_{5, l}(d)=e_{5, l}^{\prime}(c)=e_{5, l}^{\prime}(d)=0, \\
e_{5, l}(x)=e_{5, l}^{(6)}(\varepsilon) x(x-b)(x-c)^{2}(x-d)^{2} \geq 0, \\
e_{5, u}(0)=e_{5, u}(b)=e_{5, u}(c)=e_{5, l}^{\prime}(0)=e_{5, l}^{\prime}(b)=e_{5, l}^{\prime}(c)=0,
\end{gathered}
$$




$$
e_{5, u}(x)=e_{5, u}^{(6)}(\eta) x^{2}(x-b)^{2}(x-c)^{2} \leq 0,
$$

which means that $g_{5}^{l}(x) \leq \frac{\sin x}{x} \leq g_{5}^{u}(x)$. The theorem is proved.

Theorem 4 gives new two-sided bounds of $\operatorname{sinc}(x)$ using polynomials of degree 5 . The conclusion that Equation (14) achieves much tighter bounds than those of previous methods is easy to be verified. Figure 3 gives the errors between $\operatorname{sinc}(x)$ and polynomial bounds of degree 3 and 5 . However, the results of Equations (8), (9) and (14) are close in Figure 3; in particular, we give the errors between $\operatorname{sinc}(x)$ and the polynomial bounds of Equations (8), (9) and (14) in Figure 4. Figure 4 shows that Equations (8) and (9) have similar errors and the error of Equation (14) is obviously smaller than that of Equations (8) and (9).

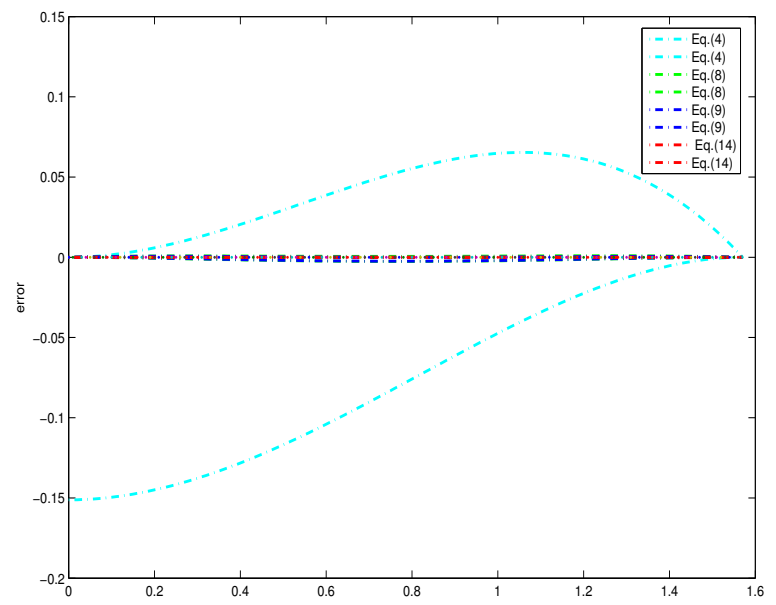

Figure 3. Error plots between $\operatorname{sinc}(x)$ and the polynomial bounds of degrees 3 and 5 .

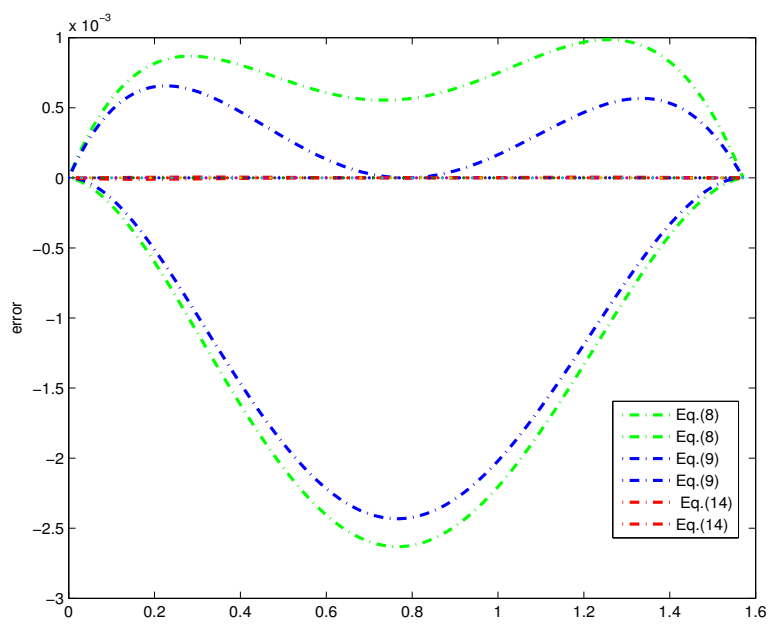

Figure 4. Error plots between sinc(x) and the polynomial bounds of Equations (8), (9) and (14).

Figure 5 gives the errors between $\operatorname{sinc}(x)$ and the polynomial bounds of degrees 4 and 5 . For the same reason, we also give the errors between $\operatorname{sinc}(x)$ and the polynomial bounds of Equations (10) and (14) in Figure 6.

Figure 7 gives a comparison between Equations (14) and (11); here, we set $m=5,8,10$ in Equation (11), where $m$ is the degree of the polynomial. Our results are obviously better than that of Zeng [15]; meanwhile, we find that the error is even greater with the increase of $m^{\prime}$ s value of Equation (11). Maximum errors of different methods are presented in Table 1. Although Equation (11) gives the polynomial bounds of degree $m$ for $\sin c(x)$, the error of Equation (11) is relatively large. 
The maximum errors of Equation (10) is close to the results of Equation (14); however, it is still very obvious that the maximum error of Equation (14) is the smallest.

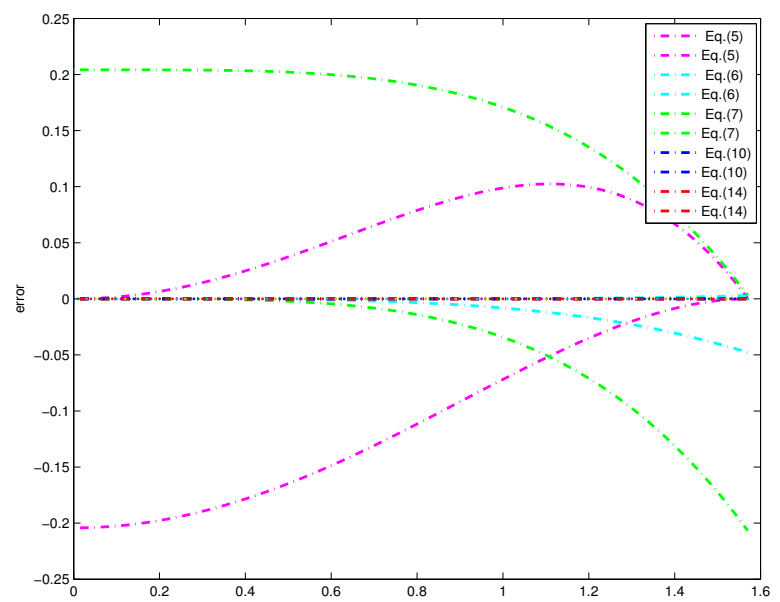

Figure 5. Error plots between $\operatorname{sinc}(x)$ and the polynomial bounds of degrees 4 and 5 .

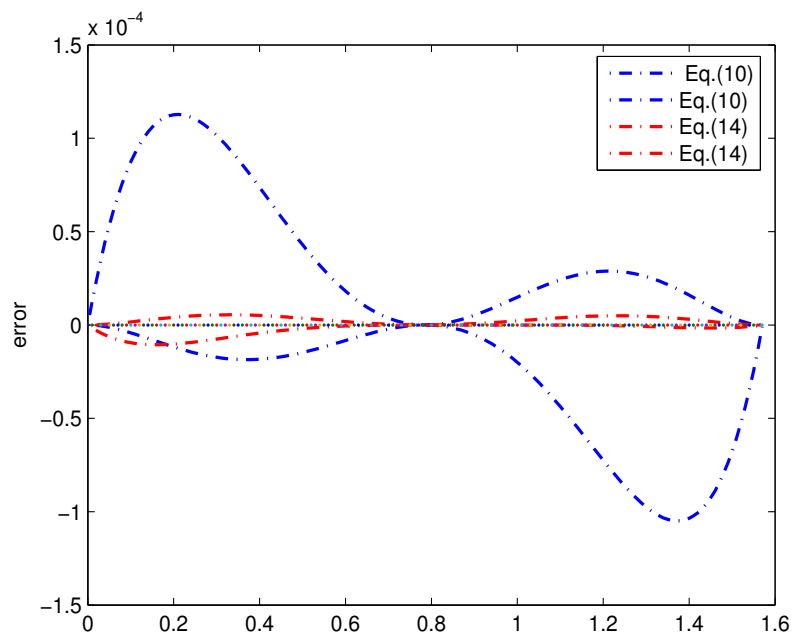

Figure 6. Error plots between $\operatorname{sinc}(x)$ and the polynomial bounds of Equations (10) and (14).

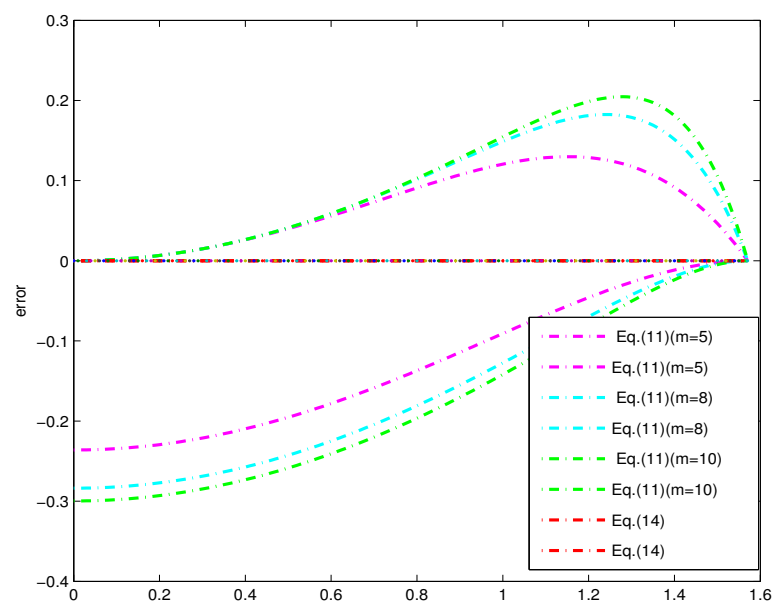

Figure 7. Error plots between sinc(x) and the polynomial bounds of Equations (11) and (14). 


\section{Conclusions}

In this paper, we gave new refinements and improvements of Jordan's inequality. Firstly, the new polynomial bounds of degrees 1 and 2 were given. The results show that our bounds are tighter than the previous results of polynomials of degrees 1 and 2. Meanwhile, we presented new improvements of Jordan's inequality using polynomials of degree 5, which can achieve much tighter bounds than those previous methods.

Much work still remains. The polynomial bounds of degree 5 were given in this paper, and the polynomial bounds of higher degree are needed for tighter bounds. However, it will require more complicated calculations. Furthermore, it is still an important problem to find tighter polynomial bounds of lower degrees.

Author Contributions: All authors contributed equally in writing this article. All authors read and approved the final manuscript.

Funding: The work is partially supported by the National Natural Science Foundation of China (No. 11701152, 11161038).

Acknowledgments: We thank the editor and referees for their careful reading and valuable suggestions to make the article reader-friendly.

Conflicts of Interest: The authors declare no conflicts of interest.

\section{References}

1. Mitrinović, D.S. Analytic Inequalities; Springer: New York, NY, USA, 1970.

2. Wu, S.; Debnath, L. A new generalized and sharp version of Jordan'S inequality and its applications to the improvement of the Yang Le inequality. Appl. Math. Lett. 2006, 19, 1378-1384. [CrossRef]

3. Zhu, L. Sharpening of Jordan's inequalities and its applications. Math. Inequal. Appl. 2006, 9, 103. [CrossRef]

4. Kuo, M.K. Refinements of Jordan's inequality. J. Inequal. Appl. 2011, 2011, 1-6. [CrossRef]

5. Chen, C.P.; Debnath, L. Sharpness and generalization of Jordan's inequality and its application. Appl. Math. Lett. 2012, 12, 594-599. [CrossRef]

6. Nishizawa, Y. Sharpening of Jordan's type and Shafer-Fink's type inequalities with exponential approximations. Appl. Math. Comput. 2015, 269, 146-154. [CrossRef]

7. Alzer, H.; Kwong, M.K. Sharp upper and lower bounds for a sine polynomial. Appl. Math. Comput. 2016, 275, 81-85. [CrossRef]

8. Zhang, X.; Wang, G.; Chu, Y. Extensions and sharpenings of Jordan's and Kober's inequalities. J. Inequal. Pure Appl. Math. 2006, 7, 98-101.

9. Qi, F.; Niu, D.W.; Guo, B.N. Refinements, Generalizations, and Applications of Jordan's Inequality and Related Problems. J. Inequal. Appl. 2009, 2009, 1-52. [CrossRef]

10. Deng, K. The noted Jordan's inequality and its extensions. J. Xiangtan Mining Inst. 1995, pp. 60-63.

11. Jiang, W.D.; Yun, H. Sharpening of Jordan's inequality and its applications. J. Inequal. Pure Appl. Math. 2006, 7, 1-8.

12. Debnath, L.; Mortici, C.; Zhu, L. Refinements of Jordan-Stečkin and Becker-Stark Inequalities. Results Math. 2015, 67, 207-215, doi:10.1007/s00025-014-0405-3. [CrossRef]

13. Agarwal, R.P.; Kim, Y.H.; Sen, S.K. A new refined Jordan's inequality and its application. Math. Inequal. Appl. 2009, 12, 255-264. [CrossRef]

14. Chen, X.D.; Shi, J.; Wang, Y.; Xiang, P. A New Method for Sharpening the Bounds of Several Special Functions. Results Math. 2017, 72, 695-702. [CrossRef]

15. Zeng, S.P.; Wu, Y.S. Some new inequalities of Joran type for sine. Sci. World J. 2013, 2013, 1-5.

16. Davis, P. Interpolation and Approximation; Dover Publications: New York, NY, USA, 1975.

(C) 2018 by the authors. Licensee MDPI, Basel, Switzerland. This article is an open access article distributed under the terms and conditions of the Creative Commons Attribution (CC BY) license (http://creativecommons.org/licenses/by/4.0/). 